\title{
COMMUTATIVE FORMAL GROUPS ARISING FROM SCHEMES
}

\author{
ANDRE CHATZISTAMATIOU
}

\begin{abstract}
We prove the following criterion for the pro-representability of the deformation cohomology of a commutative formal Lie group. Let $f$ be a flat morphism between noetherian schemes. Assume that the target of $f$ is flat over the integers. For a commutative formal Lie group $E$, we have the deformation cohomology of $f$ with coefficients in $E$ at our disposal. If the higher direct images of the tangent space of $E$ are locally free and of finite rank then the deformation cohomology is pro-representable by a commutative formal Lie group.
\end{abstract}

\section{INTRODUCTION}

Let $f: X \rightarrow S$ be a flat and separated morphism. The formal completion $\widehat{\mathrm{Pic}}_{X / S}$ of the relative Picard sheaf can be described as the first deformation cohomology $\Phi^{1}(X / S)$ with coefficients in the formal completion $\hat{\mathbb{G}}_{m}$ of the multiplicative group. Artin and Mazur, in their paper [AM77, study the higher degree analogues $\Phi^{r}(X / S)$, motivated by the idea that these sheaves exhibit a strong tendency to be pro-representable by formal Lie groups. Commutative formal Lie groups provide interesting invariants over a field of positive characteristic but poor invariants in characteristic zero where every commutative formal Lie group is isomorphic to a product of $\hat{\mathbb{G}}_{m}$.

The purpose of this paper is to give a convenient criterion for $\Phi^{r}(X / S)$ to be pro-representable when $S$ is flat over $\operatorname{Spec}(\mathbb{Z})$. In order to state our theorem we need to introduce a bit of notation. We denote by $\Phi^{r}(X / S, E)$ the deformation cohomology of a commutative formal Lie group $E$ over $X$ (see 1.2) and $\Phi^{r}(X / S)=$ $\Phi^{r}\left(X / S, \hat{\mathbb{G}}_{m}\right)$. The tangent space of $E$ is denoted by $T E$, it is a locally free $\mathcal{O}_{X^{-}}$ module; for example, $T \hat{\mathbb{G}}_{m}=\mathcal{O}_{X}$.

Theorem 1 (Theorem 3.1.1). Let $f: X \rightarrow S$ be a flat morphism between noetherian schemes. Suppose that $S$ is flat over $\operatorname{Spec}(\mathbb{Z})$. Let $E$ be a commutative formal Lie group over $X$. Suppose that $R^{r} f_{*} T E$ is locally free and of finite rank for all $r \geq r_{0}$. Then $\Phi^{r}(X / S, E)$ is pro-representable by a commutative formal Lie group over $S$ for all $r \geq r_{0}$.

In a first version of this paper, we proved Theorem 11 by using Cartier theory. It turns out that the Cartier module associated to $\Phi^{r}(X / S)$ is $H^{r}\left(X, \mathbb{W} \mathcal{O}_{X}\right)$, where $\mathbb{W} \mathcal{O}_{X}$ is the sheaf of big Witt vectors, provided that $S$ is affine. The pro-representability of $\Phi^{r}(X / S)$ can be therefore reduced to certain properties of $H^{r}\left(X, \mathbb{W} \mathcal{O}_{X}\right)$ which are made precise by Cartier theory.

This work has been supported by the SFB/TR 45 "Periods, moduli spaces and arithmetic of algebraic varieties". 
In this version of the paper, we follow a quicker and more elegant approach via Lemma 3.2.1, which was pointed out by the referee - we are very grateful to the referee for sharing this proof with us.

One motivation for the paper is the existence of a big de Rham-Witt complex $\mathbb{W} \Omega_{X}^{\bullet}$ for arbitrary schemes due to Hesselholt and Madsen [HM01, [Hes]. Here $\mathbb{W} \mathcal{O}_{X}$ appears as a quotient of $\mathbb{W} \Omega_{X}^{\bullet}$, and in view of the Bloch-Illusie slope spectral sequence for smooth varieties over a perfect field one could expect that $H^{*}\left(X, \mathbb{W} \mathcal{O}_{X}\right)$ captures an interesting part of $H^{*}\left(X, \mathbb{W} \Omega_{X}^{\bullet}\right)$.

In Section 5 we describe the relationship with the Gauss-Manin connection. If $S$ is a smooth scheme over $\mathbb{Z}$, and $\mathfrak{X}$ is a commutative formal Lie group over $S$, then the Katz-Oda construction yields a connection

$$
\nabla: H^{i}\left(\Omega_{\mathfrak{X} / S}^{*}\right) \rightarrow H^{i}\left(\Omega_{\mathfrak{X} / S}^{*}\right) \otimes_{\mathcal{O}_{S}} \Omega_{S / \mathbb{Z}}^{1} \quad \text { for all } i .
$$

Since in the invariant 1-forms (see A.1) are automatically closed, we may define $\left(H_{\text {inv }}^{1}(\mathfrak{X}), \nabla\right)$ to be the smallest subobject of $\left(H^{1}\left(\Omega_{\mathfrak{X} / S}^{*}\right), \nabla\right)$ that contains the invariant 1-forms. As an application of a theorem of Stienstra [Sti91] we will prove the following statement.

Theorem 2 (Theorem 5.3.1). Let $f: X \rightarrow S$ be a smooth, projective morphism of relative dimension $r$. Suppose that $S$ is smooth over $\operatorname{Spec}(\mathbb{Z})$ and suppose that $R^{j} f_{*} \Omega_{X / S}^{i}$ is locally free for all $i, j$. Then $\left(H_{\mathrm{inv}}^{1}\left(\Phi^{i}(X / S)\right), \nabla\right)$ is a subquotient of the Gauss-Manin connection $\left(H_{\mathrm{dR}}^{2 r-i}(X / S), \nabla\right)$ for all $i \geq 0$.

Acknowledgments. Lemma 3.2.1 and its use for proving the main theorem was shown to us by the referee for International Mathematics Research Notices. We are very grateful to the referee for providing this argument.

\section{Deformation cohomology}

1.1. Let $X$ be a scheme. Let $E$ be a sheaf of abelian groups on the big Zariski site of $X$. The formal completion $\hat{E}$ of $E$ along its zero section is defined by

$$
\hat{E}(Z):=\operatorname{ker}\left(E(Z) \rightarrow E\left(Z_{\text {red }}\right)\right) .
$$

The formal completion $\hat{E}$ is a sheaf on the big Zariski site again (cf. [AM77, II.1]).

Examples are $\hat{\mathbb{G}}_{a}$ and $\hat{\mathbb{G}}_{m}$; in both cases the sheaves are pro-represented by the formal scheme $\operatorname{Spf}\left(\mathcal{O}_{X}[|t|]\right)$, the group law being $m^{*}(t)=t \hat{\otimes} 1+1 \hat{\otimes} t$ for $\hat{\mathbb{G}}_{a}$ and $m^{*}(t)=t \hat{\otimes} 1+1 \hat{\otimes} t-t \hat{\otimes} t$ for $\hat{\mathbb{G}}_{m}$.

1.2. Let $f: X \rightarrow S$ be a morphism of schemes. For every morphism $g: T \rightarrow S$ we define a sheaf $\tilde{E}_{T}$ on the small Zariski site of $X_{T}:=X \times_{S} T$ as follows: let $\iota: X \times_{S} T_{\text {red }} \rightarrow X \times{ }_{S} T$ be the base change of $T_{\text {red }} \rightarrow T$, we set

$$
\tilde{E}_{T}:=\operatorname{ker}\left(E \rightarrow \iota_{*} E\right)
$$

i.e. $\tilde{E}_{T}(U)=\operatorname{ker}\left(E(U) \rightarrow E\left(U \times_{T} T_{\text {red }}\right)\right)$ for every open $U \subset X_{T}$. By denoting $f_{T}: X_{T} \rightarrow T$ the base change of $f$, we obtain for every integer $q \geq 0$ the sheaf $R^{q} f_{T *} \tilde{E}_{T}$ on $T$.

Definition 1.2.1. For a morphism $g: T \rightarrow S$ we set

$$
\Phi^{q}(X / S, E)(g: T \rightarrow S):=\Gamma\left(T, R^{q} f_{T *} \tilde{E}_{T}\right) .
$$


The assignment $\Phi^{q}(X / S, E)$ defines a sheaf on the big Zariski site of $S$, which is called the deformation cohomology of $X / S$ with coefficients in $E$ [AM77, II.(1.4)]. Indeed, for $T^{\prime} \stackrel{h}{\rightarrow} T \stackrel{g}{\rightarrow} S$, the natural map $\left(i d_{X} \times h\right)^{-1} \tilde{E}_{T} \rightarrow \tilde{E}_{T^{\prime}}$ induces

$$
h^{-1} R^{q} f_{T *} \tilde{E}_{T} \rightarrow R^{q} f_{T^{\prime *}}\left(i d_{X} \times h\right)^{-1} \tilde{E}_{T} \rightarrow R^{q} f_{T^{\prime} *} \tilde{E}_{T^{\prime}},
$$

equipping $\Phi^{q}(X / S, E)$ with the structure of a presheaf. Since

$$
\Gamma\left(U, R^{q} f_{T *} \tilde{E}_{T}\right)=\Gamma\left(U, R^{q} f_{U *}\left(\left.\tilde{E}_{T}\right|_{f_{T}^{-1} U}\right)\right)=\Gamma\left(U, R^{q} f_{U *} \tilde{E}_{U}\right)
$$

for every open $U$ of $T$, it is a sheaf.

For a morphism $h: Y \rightarrow X$ over $S$ we get an induced morphism of sheaves

$$
\Phi^{q}(h, E): \Phi^{q}(X / S, E) \rightarrow \Phi^{q}(Y / S, E),
$$

which is functorial in $h$.

Obviously, the deformation cohomology for $E$ and its formal completion $\hat{E}$ agree:

$$
\Phi^{q}(X / S, E)=\Phi^{q}(X / S, \hat{E}) \quad \text { for all } q .
$$

Remark 1.2.2. Artin and Mazur use the étale topology in [AM77, II] for the definition of the deformation cohomology. For the coefficients we will be considering étale and Zariski deformation cohomology agree (Lemma 1.6.1).

1.3. If $E$ is formally smooth then, by definition, we have an exact sequence

$$
0 \rightarrow \tilde{E}_{T} \rightarrow E_{\mid X_{T}} \rightarrow \iota_{*}\left(E_{\mid X_{T_{\text {red }}}}\right) \rightarrow 0 .
$$

Therefore we obtain a long exact sequence

$$
\begin{aligned}
\ldots & \rightarrow \Phi^{q}(X / S, E)_{\mid T} \rightarrow R^{q} f_{T *}\left(E_{\mid X_{T}}\right) \rightarrow R^{q} f_{T_{\mathrm{red}} *}\left(E_{\mid X_{T_{\mathrm{red}}}}\right) \rightarrow \\
& \rightarrow \Phi^{q+1}(X / S, E)_{\mid T},
\end{aligned}
$$

and $\Phi^{*}(X / S, E)$ controls the deformation of cohomology classes on $T_{\text {red }}$ to classes on $T$. A classical case is $E=\mathbb{G}_{m}$ and $S=\operatorname{Spec}(R)$ with $R$ a discrete valuation ring with uniformizer $t$ and residue field $k$. Let $T_{n}=\operatorname{Spec}\left(R / t^{n}\right)$; we get an exact sequence:

$$
\Phi^{1}\left(X / S, \hat{\mathbb{G}}_{m}\right)\left(R / t^{n}\right) \rightarrow \operatorname{Pic}\left(X_{T_{n}}\right) \rightarrow \operatorname{Pic}\left(X \otimes_{R} k\right) \rightarrow \Phi^{2}\left(X / S, \hat{\mathbb{G}}_{m}\right)\left(R / t^{n}\right) .
$$

For $L \in \operatorname{Pic}\left(X \otimes_{R} k\right)$ we obtain an obstruction class in $\lim _{n} \Phi^{2}\left(X / S, \hat{\mathbb{G}}_{m}\right)\left(R / t^{n}\right)$ which becomes trivial if $L$ lifts to $\lim _{n} \operatorname{Pic}\left(X_{T_{n}}\right)$.

1.4. Let $f: X \rightarrow S$ be a flat and proper morphism. Assume that $f$ is cohomologically flat in dimension zero. Then Pic $X / S$ is represented by an algebraic space whose formal completion along the zero section is $\Phi^{1}\left(X / S, \hat{\mathbb{G}}_{m}\right)$ (cf. [AM77, II.4]).

1.5. If $E$ is a commutative formal group over $X$ then we say that $E$ is a commutative formal Lie group if every point $x \in X$ admits an open affine neighborhood $U=\operatorname{Spec}(A)$ and an isomorphism $E \times{ }_{X} U \cong \operatorname{Spf}\left(A\left[\left|x_{1}, \ldots, x_{d}\right|\right]\right)$ as formal schemes over $\operatorname{Spec}(A)$ such that the zero $0 \in\left(E \times{ }_{X} U\right)(\operatorname{Spec}(A))$ identifies with the mor$\left.\operatorname{phism} \operatorname{Spec}(A) \rightarrow \operatorname{Spf}\left(A\left[\left|x_{1}, \ldots, x_{d}\right|\right]\right)\right)$ given by $x_{i} \mapsto 0$ for all $i$.

For a commutative formal Lie group $E$ we define the tangent space $T E$ by

$$
T E(U):=\operatorname{ker}\left(E\left(\operatorname{Spec}\left(\mathcal{O}_{U}[\epsilon] / \epsilon^{2}\right)\right) \rightarrow E(U)\right)
$$

for every open $U \subset X$. The tangent space is a locally free $\mathcal{O}_{X}$-module of finite rank. For example, $T \hat{\mathbb{G}}_{m}=\mathcal{O}_{X}=T \hat{\mathbb{G}}_{a}$. 
1.6. Again, let $f: X \rightarrow S$ be a morphism and $E$ a commutative formal Lie group over $X$. Let $g: T \rightarrow S$ be a morphism and suppose that $T=\operatorname{Spec}(R)$ for a noetherian $\operatorname{ring} R$. Set $T_{i}=\operatorname{Spec}\left(R / \operatorname{nil}(R)^{i}\right)$ for all $i \geq 1$, and

$$
\tilde{E}_{T}^{i}:=\operatorname{ker}\left(E \rightarrow \iota_{i *} E\right)
$$

with $\iota_{i}: X_{T_{i}} \rightarrow X_{T}$ the base change of $T_{i} \rightarrow T$. For an open $U \subset X_{T}$, we have $\tilde{E}_{T}^{i}(U)=\operatorname{ker}\left(E(U) \rightarrow E\left(U \times_{T} T_{i}\right)\right)$. The sheaf $\tilde{E}_{T}$ admits the filtration

$$
\tilde{E}_{T}=\tilde{E}_{T}^{1} \supset \tilde{E}_{T}^{2} \supset \cdots \supset \tilde{E}_{T}^{n}=0,
$$

provided that $\operatorname{nil}(R)^{n}=0$.

Lemma 1.6.1. For all $i \geq 1$, the quotient $\tilde{E}_{T}^{i} / \tilde{E}_{T}^{i+1}$ is a coherent $\mathcal{O}_{X_{T_{\text {red }}}}$-module. More precisely,

$$
\left(\operatorname{nil}(R)^{i} \cdot \mathcal{O}_{X_{T}} / \operatorname{nil}(R)^{i+1} \cdot \mathcal{O}_{X_{T}}\right) \otimes_{\mathcal{O}_{X_{T}}}(i d \times g)^{*} T E \stackrel{\cong}{\rightrightarrows} \tilde{E}_{T}^{i} / \tilde{E}_{T}^{i+1} .
$$

Proof. We may assume that $S=T$. There is a natural injective morphism

$$
\tilde{E}_{S}^{i} / \tilde{E}_{S}^{i+1} \rightarrow \operatorname{ker}\left(\iota_{i+1 *} E \rightarrow \iota_{i *} E\right) .
$$

Locally one easily shows that the morphism is also surjective. We have a morphism

$$
\begin{aligned}
\left(\operatorname{nil}(R)^{i} \cdot \mathcal{O}_{X} / \operatorname{nil}(R)^{i+1} \cdot \mathcal{O}_{X}\right) \otimes_{\mathcal{O}_{X}} T E & \rightarrow \operatorname{ker}\left(\iota_{i+1 *} E \rightarrow \iota_{i *} E\right) \\
n \otimes s & \mapsto \phi_{n}^{*}(s),
\end{aligned}
$$

where $\phi_{n}: X \times_{S} S_{i+1} \rightarrow \operatorname{Spec}\left(\mathcal{O}_{X}[\epsilon] / \epsilon^{2}\right)$ is defined over $X$ by $\phi_{n}^{*}(\epsilon)=n$. Again, one checks locally that the morphism is an isomorphism.

\section{Representation By a FORMAL GRoup: REDUCTION TO NiLPOTENT}

\section{ALGEBRAS}

2.1. We will be mainly concerned with the following question when $S$ is flat over $\operatorname{Spec}(\mathbb{Z})$.

Question 2.1.1. Let $E$ be a commutative formal Lie group over $X$. When is $\Phi^{q}(X / S, E)$ pro-representable by a commutative formal Lie group over $S$ ?

For simplicity we will work with noetherian schemes.

A pro-representing formal scheme $\mathfrak{X}$ with an isomorphism $\mathfrak{X} \cong \Phi^{q}(X / S, E)$ is unique up to unique isomorphism. Indeed, if $J \subset \mathcal{O}_{\mathfrak{X}}$ is the maximal ideal of definition then the scheme $X_{n}:=\left(\mathfrak{X}, \mathcal{O}_{\mathfrak{X}} / J^{n}\right)$ represents the restriction of $\Phi^{q}(X / S, E)$ to the category of schemes $T$ over $S$ such that nil $\left(\mathcal{O}_{T}\right)^{n}=0$. Thus $X_{n}$ is unique, which implies that $\mathfrak{X}=\lim _{n} X_{n}$ is unique. In particular, Question 2.1.1 is local in $S$.

2.2. Let $R$ be a commutative ring. For an $R$-algebra $A$ we define a new $R$-algebra $N_{R}(A)$ by

$$
N_{R}(A):=R \oplus \operatorname{nil}(A) /\{(r,-r) ; r \in \operatorname{nil}(R)\} .
$$

The multiplication is given by $(r, n) \cdot\left(r^{\prime}, n^{\prime}\right)=\left(r r^{\prime}, r n^{\prime}+r^{\prime} n+n n^{\prime}\right)$. Obviously,

$$
\operatorname{nil}(A) \stackrel{\cong}{\rightrightarrows} \operatorname{nil}\left(N_{R}(A)\right), \quad n \mapsto(0, n),
$$

is an isomorphism. Clearly, $N_{R}$ defines a functor

$$
N_{R}:(R \text {-algebras }) \rightarrow(R \text {-algebras }) .
$$


Moreover, there is a morphism of functors $N_{R} \rightarrow i d$ induced by the natural map

$$
N_{R}(A) \rightarrow A, \quad(r, n) \mapsto r+n .
$$

Let $\mathcal{C}$ be the full subcategory consisting of $R$-algebras $A$ such that (2.2.1) is an isomorphism. Then $N_{R}$ takes values in $\mathcal{C}$ and is right adjoined to the forgetful functor $\mathcal{C} \rightarrow(R$-algebras $)$ :

$$
\operatorname{Hom}_{R}(B, A)=\operatorname{Hom}_{R}\left(B, N_{R}(A)\right) \text { for all } B \in \mathcal{C} \text { and any } R \text {-algebra } A \text {. }
$$

Proposition 2.2.1. Let $f: X \rightarrow S=\operatorname{Spec}(R)$ be a flat morphism between noetherian schemes. Let $E$ be a commutative formal Lie group over $X$ and let $g: \operatorname{Spec}(A) \rightarrow S$ be a morphism. We assume that $A$ is noetherian. For all $q \geq 0$ there is a functorial isomorphism

$$
H^{q}\left(X_{\mathrm{Spec}\left(N_{R}(A)\right)}, \tilde{E}_{\mathrm{Spec}\left(N_{R}(A)\right)}\right) \stackrel{\cong}{\rightarrow} H^{q}\left(X_{\mathrm{Spec}(A)}, \tilde{E}_{\mathrm{Spec}(A)}\right) .
$$

Proof. By using (2.2.1) we obtain a functorial morphism (2.2.2). Set $T:=\operatorname{Spec}(A)$; for every open $U \subset X$ we claim that

$$
\Gamma\left(U_{\mathrm{Spec}\left(N_{R}(A)\right)}, \tilde{E}_{\mathrm{Spec}\left(N_{R}(A)\right)}\right) \rightarrow \Gamma\left(U_{T}, \tilde{E}_{T}\right)
$$

is an isomorphism.

In fact, we may assume that $U=X$. Suppose first that $X$ is separated. Then it is sufficient to prove (2.2.3) for every affine open $U=\operatorname{Spec}(B)$ in $X$. Moreover, we may assume that $E \times_{X} U \cong \operatorname{Spf}\left(B\left[\left|x_{1}, \ldots, x_{d}\right|\right]\right)$. Then (2.2.3) reads

$$
\prod_{i=1}^{d} \operatorname{ker}\left(B \otimes_{R} N_{R}(A) \rightarrow B \otimes_{R}\left(N_{R}(A) / \operatorname{nil}(A)\right)\right) \rightarrow \prod_{i=1}^{d} \operatorname{ker}\left(B \otimes_{R} A \rightarrow B \otimes_{R}(A / \operatorname{nil}(A))\right) \text {. }
$$

Since $B$ is flat over $R$ both sides equal $\left(B \otimes_{R} \operatorname{nil}(A)\right)^{d}$.

If $X$ is not separated then we still have (2.2.3) for every separated open $U$ of $X$. Since an open of a separated scheme is separated again, we can simply use a covering of $X$ by separated (e.g. affine) open sets to conclude (2.2.3) for $X$.

Let us prove that (2.2.2) is an isomorphism. Again, suppose first that $X$ is separated. Since (2.2.3) holds it is sufficient to show that $H^{q}\left(X_{T}, \tilde{E}_{T}\right)$, for any noetherian affine scheme $T$, can be calculated by the cohomology of the Cech complex associated to an affine open covering. This follows because $\tilde{E}_{T}$ has a finite filtration such that the graded pieces are coherent (Lemma 1.6.1).

If $X$ is not separated then we take a finite covering $\mathfrak{U}$ of $X$ by affine open sets. By using the spectral sequence

$$
E_{1}^{p, q}=\bigoplus_{i_{0}<\cdots<i_{p}} H^{q}\left(\bigcap_{j=0}^{p} U_{i_{j}, T}, \tilde{E}_{T}\right) \Rightarrow H^{p+q}\left(X_{T}, \tilde{E}_{T}\right),
$$

we reduce to the statement for the separated schemes $\bigcap_{j=0}^{p} U_{i_{j}}$.

2.3. Let $R$ be a commutative algebra. By a nilpotent $R$-algebra $\mathcal{N}$ we mean an $R$-algebra $\mathcal{N}$ without 1-element, such that $\mathcal{N}^{r}=0$ for an integer $r \geq 1$. There is an obvious $R$-algebra $R \oplus \mathcal{N}$ attached to every nilpotent $R$-algebra $\mathcal{N}$.

Let $f: X \rightarrow \operatorname{Spec}(R)$ be a morphism and $E$ a commutative formal Lie group over $X$. For a nilpotent $R$-algebra $\mathcal{N}$ we define a sheaf $\tilde{E}_{\mathcal{N}}$ on the small Zariski site of $X_{\operatorname{Spec}(R \oplus \mathcal{N})}$ by

$$
\tilde{E}_{\mathcal{N}}=\operatorname{ker}\left(\tilde{E}_{\mathrm{Spec}(R \oplus \mathcal{N})} \rightarrow \iota_{*} \tilde{E}_{\mathrm{Spec}(R)}\right),
$$


where $\iota: \operatorname{Spec}(R) \rightarrow \operatorname{Spec}(R \oplus \mathcal{N})$ is induced by $R \oplus \mathcal{N} \rightarrow R,(r, n) \mapsto r$. We note that $X \rightarrow X_{\mathrm{Spec}(R \oplus \mathcal{N})}$ is topologically an isomorphism, and we can consider $\tilde{E}_{\mathcal{N}}$ as sheaf on $X$.

For an integer $q \geq 0$ we define

$$
\begin{aligned}
\Psi^{q}(X / S, E):(\text { nilpotent } R \text {-algebras }) & \rightarrow \text { (abelian groups) } \\
\Psi^{q}(X / S, E)(\mathcal{N}) & =H^{q}\left(X, \tilde{E}_{\mathcal{N}}\right) .
\end{aligned}
$$

Notation 2.3.1. We say that a functor

$$
\Psi: \text { (nilpotent } R \text {-algebras) } \rightarrow \text { (abelian groups) }
$$

is pro-represented by the formal group $\mathfrak{X}$ over $R$ if

$$
\Psi \cong[\mathcal{N} \mapsto \operatorname{ker}(\mathfrak{X}(R \oplus \mathcal{N}) \rightarrow \mathfrak{X}(R))]
$$

Proposition 2.3.2. Let $q \geq 0$ be an integer. Let $f: X \rightarrow S=\operatorname{Spec}(R)$ be a flat morphism between noetherian schemes. Let $E$ be a commutative formal Lie group over $X$. If $\Psi^{q}(X / S, E)$ is pro-represented by a commutative formal Lie group $\mathfrak{X}$ (over $S$ ) then the restriction of $\Phi^{q}(X / S, E)$ to the category of noetherian schemes (over $S$ ) is pro-represented by $\mathfrak{X}$.

Proof. For a noetherian $R$-algebra $A$ we have functorial isomorphisms

$$
\Psi^{q}(X / S, E)(\operatorname{nil}(A)) \cong H^{q}\left(X_{\operatorname{Spec}\left(N_{R}(A)\right)}, \tilde{E}\right) \stackrel{\cong}{\rightrightarrows} H^{q}\left(X_{\operatorname{Spec}(A)}, \tilde{E}\right) .
$$

The first isomorphism is obvious and the second follows from Proposition 2.2.1 On the other hand, $\operatorname{ker}(\mathfrak{X}(R \oplus \operatorname{nil}(A)) \rightarrow \mathfrak{X}(R)) \cong \mathfrak{X}(A)$. Therefore the functor

$$
(R \text {-alg }) \rightarrow(\text { abelian groups }), \quad A \mapsto H^{q}\left(X_{\operatorname{Spec}(A)}, \tilde{E}_{\operatorname{Spec}(A)}\right),
$$

is pro-represented by $\mathfrak{X}$.

We have a morphism of functors

$$
\left[A \mapsto H^{q}\left(X_{\operatorname{Spec}(A)}, \tilde{E}\right)\right] \rightarrow\left[A \mapsto \Phi^{q}(X / S, E)(\operatorname{Spec}(A))\right],
$$

and the right hand side is the sheafication of the left hand side. Since $\mathfrak{X}$ defines a sheaf, the restriction of $\Phi^{q}(X / S, E)$ to the category of noetherian affine schemes is represented by $\mathfrak{X}$. Again, since $\Phi^{q}(X / S, E)$ and $\mathfrak{X}$ are both sheaves we obtain $\Phi^{q}(X / S, E) \cong \mathfrak{X}$.

As a criterion for pro-representability by a formal Lie group we will use the following proposition.

Proposition 2.3.3. [Zin84, 2.32] Let $H:$ (nilpotent $R$-algebras) $\rightarrow$ (ab. groups) be an exact functor which commutes with arbitrary direct sums. Suppose that $H\left(x R[x] / x^{2} R[x]\right)$ is a free and finitely generated $R$-module. Then $H$ is represented by a commutative formal Lie group $\mathfrak{X}$ in the sense of Notation 2.3.1.

Remark 2.3.4. A short exact sequence in the category of nilpotent $R$-algebras is by definition a sequence $0 \rightarrow N_{1} \rightarrow N_{2} \rightarrow N_{3} \rightarrow 0$ of morphisms of nilpotent $R$ algebras that is exact as a sequence of $R$-modules. A functor $H$ as above is called exact if it preserves exact sequences. Left and right exact functors are defined in the same way.

By definition, a functor $H$ commutes with arbitrary direct sums if the natural map

$$
\bigoplus_{i \in I} H\left(N_{I}\right) \rightarrow H\left(\bigoplus_{i \in I} N_{i}\right)
$$


is an isomorphism for all sets $\left\{N_{i}\right\}_{i \in I}$ of nilpotent $R$-algebras with the property that there exists $k \geq 1$ with $N_{i}^{k}=0$ for all $i \in I$.

\section{REPRESENTATION BY FORMAL GROUPS}

3.1. Formulation of the main theorem. Let $f: X \rightarrow S$ be a morphism of schemes. Let $E$ be a commutative formal Lie group over $X$ (see 1.5), e.g. $E=\hat{\mathbb{G}}_{m}$, the formal completion of $\mathbb{G}_{m}$ (see 1.1). The tangent space of $E$ is denoted by $T E$. Recall Definition 1.2.1 for the Artin-Mazur functor $\Phi^{q}(X / S, E)$.

Theorem 3.1.1. Let $f: X \rightarrow S$ be a flat morphism between noetherian schemes. Suppose that $S$ is flat over $\operatorname{Spec}(\mathbb{Z})$. Let $E$ be a commutative formal Lie group over $X$. Suppose that $R^{q} f_{*} T E$ is locally free and of finite rank for all $q \geq q_{0}$. Then the restriction of $\Phi^{q}(X / S, E)$ to the category of noetherian schemes (over $S$ ) is pro-representable by a commutative formal Lie group with tangent space $R^{q} f_{*} T E$ for all $q \geq q_{0}$.

3.2. Proof of the main theorem. The following lemma on which the proof is based, was shown to us by the referee for International Mathematics Research Notices.

Lemma 3.2.1. Let $R$ be a $\mathbb{Z}$-torsion free ring. Let

$$
\Phi: \text { (nilpotent } R \text {-algebras) } \rightarrow \text { (abelian groups) }
$$

be a right exact functor. Assume that the restriction of $\Phi$ to the following two categories is exact:

(1) (nilpotent $R \otimes \mathbb{Q}$-algebras),

(2) (nilpotent $R$-algebras $N$ such that $N^{2}=0$ ).

Then $\Phi$ is exact.

Proof. It suffices to show that $\Phi$ preserves injective maps of nilpotent $R$-algebras. We remark that an exact functor preserves injective maps [Zin84, Theorem 2.16].

Step 1. We claim that $\Phi(N) \rightarrow \Phi\left(N \otimes_{\mathbb{Z}} \mathbb{Q}\right)$ is injective if $N$ is $\mathbb{Z}$-torsion-free. By assumption the claim holds if $N^{2}=0$. For the general case, set $N_{\mathbb{Q}}:=N \otimes_{\mathbb{Z}} \mathbb{Q}$ and define $N^{\prime}:=N_{\mathbb{Q}}^{2} \cap N$. Note that $N^{\prime} \subset N$ is an ideal and $N / N^{\prime}$ is $\mathbb{Z}$-torsion-free. We obtain a diagram of exact sequences

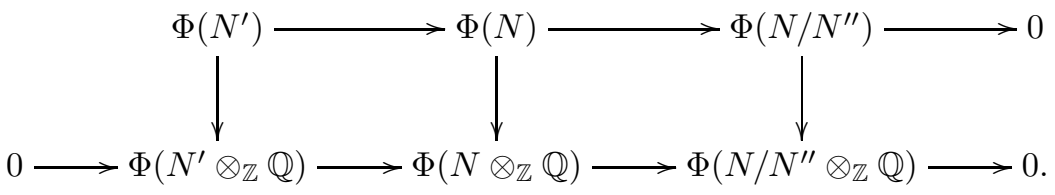

The left and right vertical arrows are injective by induction on the exponent that annihilates the nilpotent algebra. Hence the central vertical arrow is injective.

Step 2. If $N^{\prime} \rightarrow N$ is an injective morphism of nilpotent $R$-algebras and $N^{\prime}$ is $\mathbb{Z}$-torsion-free then $\Phi\left(N^{\prime}\right) \rightarrow \Phi(N)$ is injective. Indeed, the first arrow in

$$
\Phi\left(N^{\prime}\right) \rightarrow \Phi\left(N^{\prime} \otimes_{\mathbb{Z}} \mathbb{Q}\right) \rightarrow \Phi\left(N \otimes_{\mathbb{Z}} \mathbb{Q}\right)
$$

is injective by using the first step, and the second arrow is injective by assumption.

Step 3. Let $N^{\prime} \rightarrow N$ be any injective morphism of nilpotent $R$-algebras. There exists a surjective morphism of nilpotent $R$-algebras $T \rightarrow N$ such that $T$ is a free $R$-module. Let $T^{\prime}:=N^{\prime} \times{ }_{N} T$ be the fiber product, and let $I=\operatorname{ker}(T \rightarrow N)$. Since 
$R$ is $\mathbb{Z}$-torsion-free, $T^{\prime}$ and $I$ are $\mathbb{Z}$-torsion-free. By using the second step we obtain a morphism of exact sequences

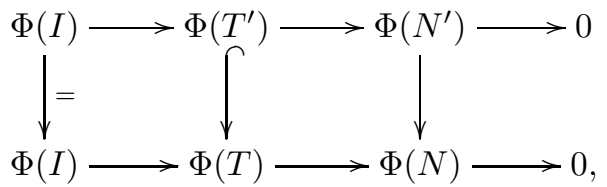

where the central vertical arrow is injective. This implies that $\Phi\left(N^{\prime}\right) \rightarrow \Phi(N)$ is injective.

Proof of Theorem 3.1.1. In view of the uniqueness of a pro-representing formal group we may assume that $S=\operatorname{Spec}(R)$ is affine. By Proposition 2.3.2 it suffices to show that $\Psi^{q}(X / S, E)$ is pro-representable by a commutative formal Lie group (see (2.3.1) for the definition of $\Psi^{q}(X / S, E)$ ).

Suppose $N$ is a nilpotent $R$-algebra such that $N^{2}=0$. Then

$$
\Psi^{q}(X / S, E)(N)=H^{q}\left(X, T E \otimes_{R} N\right),
$$

and our assumptions imply $H^{q}\left(X, T E \otimes_{R} N\right)=H^{q}(X, T E) \otimes_{R} N$ for all $q \geq q_{0}$. In particular, the restriction of $\Psi^{q}(X / S, E)$ to the category of nilpotent $R$-algebras with vanishing square is exact if $q \geq q_{0}$.

Let us denote by $S_{\mathbb{Q}}=S \times_{\operatorname{Spec}(\mathbb{Z})} \operatorname{Spec}(\mathbb{Q})$ the base change. For any nilpotent $R \otimes_{\mathbb{Z}} \mathbb{Q}$-algebra $N$ we have

$$
\Psi^{q}(X / S, E)(N)=\Psi^{q}\left(X_{\mathbb{Q}} / S_{\mathbb{Q}}, E_{\mathbb{Q}}\right)(N) \cong H^{q}\left(X_{\mathbb{Q}}, T E_{\mathbb{Q}} \otimes_{R \otimes \mathbb{Q}} N\right),
$$

because there is an isomorphism $E_{\mathbb{Q}} \cong \widehat{T E}_{\mathbb{Q}}$ (see A.2). As above we conclude that the restriction of $\Psi^{q}(X / S, E)$ to the category of nilpotent $R \otimes \mathbb{Q}$-algebras is exact if $q \geq q_{0}$.

In view of Lemma 3.2.1 we need to show that $\Psi^{q}(X / S, E)$, for $q \geq q_{0}$, is right exact in order to conclude that $\Psi^{q}(X / S, E)$ is exact. We will prove this by descending induction on $q$. Indeed, since $X / S$ is flat, every short exact sequence $0 \rightarrow N_{1} \rightarrow N_{2} \rightarrow N_{3} \rightarrow 0$ of nilpotent $R$-algebras gives rise to a long exact sequence:

$$
\begin{aligned}
\ldots \rightarrow \Psi^{q}(X / S, E)\left(N_{1}\right) \rightarrow \Psi^{q}(X / S, E)\left(N_{2}\right) \rightarrow \Psi^{q}(X / S, E)\left(N_{3}\right) \rightarrow & \\
& \Psi^{q+1}(X / S, E)\left(N_{1}\right) \rightarrow \ldots,
\end{aligned}
$$

and we know $\Psi^{q}(X / S, E)=0$ for $q>\operatorname{dim} X$.

Finally, in order to apply Proposition 2.3.3. we have to show that $\Psi^{q}(X / S, E)$, for $q \geq q_{0}$, commutes with arbitrary direct sums. Since $\Psi^{q}(X / S, E)$ is exact it is sufficient to consider the restriction to nilpotent $R$-algebras $N$ with $N^{2}=0$, where the statement is evident.

\section{Deformation cohomology of $\mathbb{G}_{m}$ and Witt vector cohomology}

4.1. Cartier modules. Let $R$ be a commutative ring. For a functor

$$
F:(\text { nilpotent } R \text {-algebras }) \rightarrow \text { (abelian groups), }
$$

we set

$$
F(x R[|x|]):={\underset{\varliminf}{n}}_{\lim _{n}} F\left(x R[x] / x^{n} R[x]\right)
$$


The assignment $C: F \mapsto F(x R[|x|])$ is functorial. For a morphism $\Phi: F \rightarrow G$ of functors we denote by $\Phi_{R[|x|]}: F(x R[|x|]) \rightarrow G(x R[|x|])$ the induced morphism.

Define the functor $\Lambda$ by

$$
\begin{gathered}
\Lambda: \text { (nilpotent } R \text {-algebras) } \rightarrow \text { (abelian groups), } \\
\Lambda(\mathcal{N}):=\left\{1+n_{1} t+n_{2} t^{2}+\cdots+n_{r} t^{r} \mid r \geq 0, n_{i} \in \mathcal{N}\right\} \subset(R \oplus \mathcal{N})[t]^{\times} .
\end{gathered}
$$

Obviously, $\Lambda$ is exact.

Theorem 4.1.1. Zin84, 3.5] Let $H$ be a left exact functor. The morphism of abelian groups

$$
\operatorname{Hom}(\Lambda, H) \stackrel{\cong}{\rightarrow} H(x R[|x|]), \quad \Phi \mapsto \Phi_{R[|x|]}(1-x t),
$$

is an isomorphism, where $1-x t \in \Lambda(x R[|x|])$.

In the theorem, $\operatorname{Hom}(\Lambda, H)$ denotes the natural transformations from $\Lambda$ to $H$.

Definition 4.1.2 (Cartier ring). Let $R$ be a commutative ring. We set

$$
\mathbb{E}_{R}:=\operatorname{End}(\Lambda)^{o p},
$$

where (.) ${ }^{o p}$ denotes the opposite ring; $\mathbb{E}_{R}$ is called the Cartier ring.

Theorem 4.1.1 implies immediately that $H(x R[|x|])$ comes equipped with a natural left $\mathbb{E}_{R}$-module structure; $H(x R[|x|])$ is called the Cartier module of $H$.

In view of

we obtain the following elements in $\mathbb{E}_{R}$ :

$$
\mathbb{E}_{R}^{o p}=\operatorname{End}(\Lambda) \stackrel{\cong, \lambda}{\longrightarrow} \Lambda(x R[|x|]),
$$

$$
\begin{aligned}
V_{n}:=\lambda^{-1}\left(1-x^{n} t\right), & F_{n}:=\lambda^{-1}\left(1-x t^{n}\right), & & \text { for } n \geq 1, \\
& {[c]:=\lambda^{-1}(1-c x t), } & & \text { for } c \in R .
\end{aligned}
$$

Theorem 4.1.3. [Zin84, 3.12] Every element $\xi \in \mathbb{E}_{R}$ has a unique representation

$$
\xi=\sum_{n, m \geq 1} V_{n}\left[a_{n, m}\right] F_{m},
$$

with $a_{n, m} \in R$, and for every fixed $n$ almost all $a_{n, m}$ vanish; in other words, $a_{n, m}=$ 0 for $m \geq m_{0}(n)$ with $m_{0}(n)$ depending on $n$.

Proposition 4.1.4. Zin84, 3.13] The following relations hold in $\mathbb{E}_{R}$ :

$$
\begin{array}{llr}
F_{1}=1=V_{1} & F_{n} V_{n}=n & \text { for } n \geq 1, \\
{[c] V_{n}=V_{n}\left[c^{n}\right]} & F_{n}[c]=\left[c^{n}\right] F_{n} & \text { for } n \geq 1, c \in R, \\
V_{m} V_{n}=V_{n \cdot m} & F_{n} F_{m}=F_{n \cdot m} & \text { for } m, n \geq 1, \\
& F_{n} V_{m}=V_{m} F_{n} & \text { if } \operatorname{gcd}(m, n)=1 .
\end{array}
$$

For $c_{1}, c_{2} \in R$ we have

$$
\begin{aligned}
{\left[c_{1}\right]\left[c_{2}\right] } & =\left[c_{1} c_{2}\right] \\
{\left[c_{1}+c_{2}\right] } & =\left[c_{1}\right]+\left[c_{2}\right]+\sum_{n=2}^{\infty} V_{n}\left[a_{n}\left(c_{1}, c_{2}\right)\right] F_{n},
\end{aligned}
$$

with $a_{n} \in \mathbb{Z}\left[X_{1}, X_{2}\right]$.

The subset $\left\{\sum_{n \geq 1} V_{n}\left[c_{n}\right] F_{n} \mid \forall n: c_{n} \in R\right\}$ of $\mathbb{E}_{R}$ defines a subring $\mathbb{W}(R)$ and is called the ring of big Witt vectors of $R$. 
Example 4.1.5. We have an isomorphism of $\mathbb{E}_{R}$-modules

$$
\begin{gathered}
\mathbb{E}_{R} / \mathbb{E}_{R} \cdot\left(F_{n}-1 \mid n \in \mathbb{Z}_{\geq 2}\right) \rightarrow \hat{\mathbb{G}}_{m}(x R[|x|]) \\
1 \mapsto 1-x, \quad V_{n} \mapsto 1-x^{n}, \quad[c] \mapsto 1-c x .
\end{gathered}
$$

In other words, the Cartier module of $\hat{\mathbb{G}}_{m, R}$ is $\mathbb{E}_{R}$ modulo the (left) ideal generated by the elements $F_{n}-1$ where $n$ runs through all natural numbers. Via

$$
\mathbb{W}(R) \rightarrow \mathbb{E}_{R} / \mathbb{E}_{R} \cdot\left(F_{n}-1 \mid n \in \mathbb{Z}_{\geq 2}\right) \rightarrow \hat{\mathbb{G}}_{m}(x R[|x|])
$$

we obtain an isomorphism $\mathbb{W}(R) \cong \hat{\mathbb{G}}_{m}(x R[|x|])$ of $\mathbb{W}(R)$-modules.

We define

$$
\mathbb{W}_{[1, n]}(R):=\hat{\mathbb{G}}_{m}\left(x R[x] / x^{n+1} R[x]\right)=\left\{1+\sum_{i=1}^{n} b_{i} x^{i} \mid b_{i} \in R\right\} .
$$

For the Cartier module of $\hat{\mathbb{G}}_{a}$ we have an isomorphism

$$
\begin{gathered}
\mathbb{E}_{R} / \mathbb{E}_{R} \cdot\left(F_{n} \mid n \in \mathbb{Z}_{\geq 2}\right) \rightarrow \hat{\mathbb{G}}_{a}(x R[|x|]) \\
1 \mapsto x, \quad V_{n} \mapsto x^{n}, \quad[c] \mapsto c x .
\end{gathered}
$$

More explicitly, with the identification $\hat{\mathbb{G}}_{a}(x R[|x|])=x R[|x|]$, we get

$$
V_{n}(g(x))=g\left(x^{n}\right), \quad F_{n}\left(c x^{i}\right)=\left\{\begin{array}{ll}
n c x^{\frac{i}{n}} & \text { if } n \text { divides } i, \\
0 & \text { otherwise, }
\end{array} \quad[c](g(x))=g(c x) .\right.
$$

Proposition 4.1.6. Let $f: X \rightarrow \operatorname{Spec}(R)$ be a flat separated morphism between noetherian schemes. We have

$$
\Psi^{q}\left(X / S, \hat{\mathbb{G}}_{m}\right)(x R[|x|])={\underset{\varliminf}{n}}_{\lim _{n}} H^{q}\left(X, \mathbb{W}_{[1, n]} \mathcal{O}_{X}\right) .
$$

Proof. We need to show $\Psi^{q}\left(X / S, \hat{\mathbb{G}}_{m}\right)\left(x R[x] / x^{n+1} R[x]\right)=H^{q}\left(X, \mathbb{W}_{[1, n]} \mathcal{O}_{X}\right)$.

Let $\left\{U_{i}\right\}_{i=1, \ldots, n}$ be an affine covering of $X$. We set $A_{J}=\Gamma\left(\bigcap_{k=0}^{s} U_{J_{k}}, \mathcal{O}_{X}\right)$ for all $J=\left(\jmath_{0}<\cdots<\jmath_{s}\right)$. Set $E=\hat{\mathbb{G}}_{m}$; if $\mathcal{N}$ is a nilpotent $R$-algebra then Lemma 1.6.1 implies that

$$
H^{q}\left(X, \tilde{E}_{\mathcal{N}}\right)=H^{q}\left(\mathcal{C}^{*}\left(\left\{U_{i}\right\}, \tilde{E}_{\mathcal{N}}\right)\right)
$$

where $\mathcal{C}^{*}\left(\left\{U_{i}\right\}, \tilde{E}_{\mathcal{N}}\right)$ is the Čech complex. Explicitly, we have

$$
\mathcal{C}^{p}\left(\left\{U_{i}\right\}, \tilde{E}_{x R[x] / x^{n+1} R[x]}\right)=\bigoplus_{|J|=p+1} \mathbb{W}_{[1, n]}\left(A_{J}\right) .
$$

Since the cohomology of $\mathbb{W}_{[1, n]}\left(\mathcal{O}_{X}\right)$ is also computed by using the Cech complex, we are done.

Proposition 4.1.7. Let $f: X \rightarrow S$ be a flat separated morphism between noetherian schemes. Suppose that $S=\operatorname{Spec}(R)$ is flat over $\operatorname{Spec}(\mathbb{Z})$. Suppose that $R^{q} f_{*} \mathcal{O}_{X}$ is locally free and of finite rank for all $q \geq q_{0}$. The Cartier module of $\Phi^{q_{0}}\left(X / S, \hat{\mathbb{G}}_{m}\right)$ is given by $H^{q_{0}}\left(X, \mathbb{W} \mathcal{O}_{X}\right)$ where $\mathbb{W} \mathcal{O}_{X}$ is the sheaf of big Witt vectors.

Proof. Proposition 4.1.6 implies that we have to show

$$
H^{q_{0}}\left(X, \mathbb{W} \mathcal{O}_{X}\right)=\underbrace{\lim _{n}}_{n} H^{q_{0}}\left(X, \mathbb{W}_{[1, n]} \mathcal{O}_{X}\right) .
$$


This follows from $R^{1} \lim _{n} H^{q_{0}-1}\left(X, \mathbb{W}_{[1, n]} \mathcal{O}_{X}\right)=0$. By using the long exact sequence for $\Psi^{*}\left(X / S, \hat{\mathbb{G}}_{m}\right)$, Theorem 3.1.1 implies that $\Psi^{q_{0}-1}\left(X / S, \hat{\mathbb{G}}_{m}\right)$ is right exact. Therefore the transition maps $H^{q_{0}-1}\left(X, \mathbb{W}_{[1, n+1]} \mathcal{O}_{X}\right) \rightarrow H^{q_{0}-1}\left(X, \mathbb{W}_{[1, n]} \mathcal{O}_{X}\right)$ are surjective.

\section{Gauss-Manin connection}

5.1. Let $S$ be a smooth scheme over $\operatorname{Spec}(\mathbb{Z})$. Let $\mathfrak{X}$ be a commutative formal Lie group over $S$. We have the de-Rham complex $\Omega_{\mathfrak{X} / S}^{*}$ at disposal and the Katz-Oda KO68 construction yields an integrable connection

$$
\nabla: H^{i}\left(\Omega_{\mathfrak{X} / S}^{*}\right) \rightarrow H^{i}\left(\Omega_{\mathfrak{X} / S}^{*}\right) \otimes_{\mathcal{O}_{S}} \Omega_{S / \mathbb{Z}}^{1} \quad \text { for all } i .
$$

Locally, we can write a closed $i$-form $\omega$ as

$$
\omega=\sum_{J=\left(j_{1}, \ldots, j_{i}\right)} \omega_{J} \cdot d x_{j_{1}} \wedge \cdots \wedge d x_{j_{i}}
$$

with $\omega_{J}=\sum_{K=\left(k_{1}, \ldots, k_{d}\right)} a_{J, K} \cdot x_{1}^{k_{1}} \cdots x_{d}^{k_{d}}$, and $a_{J, K} \in \mathcal{O}_{S}$. For a section $\xi \in T_{S / \mathbb{Z}}$ the action is simply given by

$$
\nabla_{\xi}(\omega)=\sum_{J=\left(j_{1}, \ldots, j_{i}\right)} \sum_{K=\left(k_{1}, \ldots, k_{d}\right)} \xi\left(a_{J, K}\right) \cdot x_{1}^{k_{1}} \cdots x_{d}^{k_{d}} \cdot d x_{j_{1}} \wedge \cdots \wedge d x_{j_{i}} .
$$

In view of Proposition A.1.3 we have a morphism

$$
\eta: T \mathfrak{X}^{\vee} \rightarrow \Omega_{\mathfrak{X} / S}^{1},
$$

inducing an isomorphism with the invariant 1-forms. By using Lemma A.1.4 we obtain

$$
\bar{\eta}: T \mathfrak{X}^{\vee} \rightarrow H^{1}\left(\Omega_{\mathfrak{X} / S}^{*}\right) .
$$

Definition 5.1.1. We define $H_{\text {inv }}^{1}(\mathfrak{X}) \subset H^{1}\left(\Omega_{\mathfrak{X} / S}^{*}\right)$ to be the smallest $\mathcal{O}_{S}$-submodule such that $H_{\mathrm{inv}}^{1}(\mathfrak{X})$ contains the image of $\bar{\eta}$, and the connection on $H^{1}\left(\Omega_{\mathfrak{X} / S}^{*}\right)$ induces a connection on $H_{\mathrm{inv}}^{1}(\mathfrak{X})$.

5.2. It is more convenient to work with modules rather than with connections. We denote by $D_{S}$ the sheaf differential operators and we denote by $D_{S}^{\prime}$ the subsheaf generated (as $\mathcal{O}_{S^{-}}$-algebra) by differential operators of order $\leq 1$. Locally, we can write $S=\operatorname{Spec}(R)$ and suppose that there is an étale morphism $\mathbb{Z}\left[x_{1}, \ldots, x_{n}\right] \rightarrow R$. As an $R$-module we have

$$
D_{S}^{\prime}(S)=R \otimes_{\mathbb{Z}} \mathbb{Z}\left[\partial_{1}, \ldots, \partial_{n}\right]
$$

with $\partial_{i}\left(x_{j}\right)=\delta_{i, j}$, the ring structure being uniquely determined by

$$
\partial_{i} \partial_{j}=\partial_{j} \partial_{i}, \quad \partial_{i} r=r \partial_{i}+\partial_{i}(r) \quad \text { for all } i, j \text { and } r \in R .
$$

We have an obvious equivalence of categories between $\mathcal{O}_{S}$-modules with integrable connection and $D_{S}^{\prime}$-modules. By definition, $H_{\mathrm{inv}}^{1}(\mathfrak{X})$ is the $D_{S^{-}}^{\prime}$-submodule of $H^{1}\left(\Omega_{\mathfrak{X} / S}^{*}\right)$ generated by $\bar{\eta}\left(T \mathfrak{X}^{\vee}\right)$, thus it comes equipped with a surjective morphism

$$
D_{S}^{\prime} \otimes_{\mathcal{O}_{S}} T \mathfrak{X}^{\vee} \rightarrow H_{\text {inv }}^{1}(\mathfrak{X}) .
$$

In particular, $H_{\mathrm{inv}}^{1}(\mathfrak{X})$ is a quasi-coherent $\mathcal{O}_{S}$-module. 
5.3. Let $f: X \rightarrow S$ be a smooth, projective morphism of relative dimension $r$. As above we assume that $S$ is smooth over $\operatorname{Spec}(\mathbb{Z})$. The de-Rham cohomology

$$
H_{\mathrm{dR}}^{i}(X / S):=R^{i} f_{*}\left(\Omega_{X / S}^{*}\right)
$$

comes equipped with the Gauss-Manin connection, hence defines a $D_{S}^{\prime}$-module.

Theorem 5.3.1. Let $f: X \rightarrow S$ be a smooth, projective morphism of relative dimension $r$. Suppose that $S$ is smooth over $\operatorname{Spec}(\mathbb{Z})$ and suppose that $R^{j} f_{*} \Omega_{X / S}^{i}$ is locally free for all $i, j$. Then $H_{\mathrm{inv}}^{1}\left(\Phi^{i}(X / S)\right)$ is a subquotient of $H_{\mathrm{dR}}^{2 r-i}(X / S)$ as $D_{S}^{\prime}$-module for all $i \geq 0$.

In fact, it will be easier to prove the theorem when stated in a more precise way (Theorem 5.3.3). Theorem 3.1.1 guarantees the pro-representability of $\Phi^{i}(X / S)=$ $\Phi^{i}\left(X / S, \hat{\mathbb{G}}_{m}\right)$. Let $X_{\mathbb{Q}}$ and $S_{\mathbb{Q}}$ denote the base change to $\operatorname{Spec}(\mathbb{Q})$. Obviously, we have

$$
H_{\mathrm{dR}}^{2 r-i}(X / S) \otimes_{\mathcal{O}_{S}} \mathcal{O}_{S_{\mathbb{Q}}}=H_{\mathrm{dR}}^{i}\left(X_{\mathbb{Q}} / S_{\mathbb{Q}}\right)
$$

Hence, Theorem 5.3.1 implies the following corollary.

Corollary 5.3.2. Let $f: X \rightarrow S$ be a smooth, projective morphism of relative dimension $r$. Suppose that $S$ is smooth over $\operatorname{Spec}(\mathbb{Z})$ and suppose that $R^{j} f_{*} \mathcal{O}_{X}$ is locally free for all $j$. Then, for all $i$, the sheaf $H_{\mathrm{inv}}^{1}\left(\Phi^{i}(X / S)\right) \otimes_{\mathcal{O}_{S}} \mathcal{O}_{S_{\mathbb{Q}}}$ is a subquotient of $H_{\mathrm{dR}}^{2 r-i}\left(X_{\mathbb{Q}} / S_{\mathbb{Q}}\right)$ as module over the sheaf of differential operators of $S_{\mathbb{Q}}$.

Proof. We know that $R^{j} f_{*} \Omega_{X_{\mathbb{Q}} / S_{\mathbb{Q}}}^{i}$ is locally free by Hodge theory. Thus there exists an integer $n \neq 0$ such that the base change of $f$ to $S_{\mathbb{Z}\left[n^{-1}\right]}=S \times_{\operatorname{Spec}(\mathbb{Z})} \operatorname{Spec}\left(\mathbb{Z}\left[n^{-1}\right]\right)$ satisfies the assumptions in Theorem 5.3.1. Since

$$
H_{\text {inv }}^{1}\left(\Phi^{i}\left(X_{\mathbb{Z}\left[n^{-1}\right]} / S_{\mathbb{Z}\left[n^{-1}\right]}\right)\right) \otimes_{\mathcal{O}_{S_{\mathbb{Z}\left[n^{-1}\right]}}} \mathcal{O}_{S_{\mathbb{Q}}}=H_{\text {inv }}^{1}\left(\Phi^{i}(X / S)\right) \otimes_{\mathcal{O}_{S}} \mathcal{O}_{S_{\mathbb{Q}}}
$$

the statement follows.

Of course, $H_{\text {inv }}^{1}\left(\Phi^{i}\left(X_{\mathbb{Q}} / S_{\mathbb{Q}}\right)\right)$ vanishes. But $H_{\text {inv }}^{1}\left(\Phi^{i}(X / S)\right) \otimes_{\mathcal{O}_{S}} \mathcal{O}_{S_{\mathbb{Q}}}$ is in general non-trivial (see Examples 5.4.2).

Since $f$ is of relative dimension $r$, we get a natural morphism

$$
\epsilon: R^{r-i} f_{*} \Omega_{X / S}^{r} \rightarrow H_{\mathrm{dR}}^{2 r-i}(X / S) .
$$

Therefore, $D_{S}^{\prime} \cdot \epsilon\left(R^{r-i} f_{*} \Omega_{X / S}^{r}\right)$ is a $D_{S}^{\prime}$-submodule of $H_{\mathrm{dR}}^{2 r-i}(X / S)$. Moreover, Grothendieck duality implies the existence of a canonical morphism

$$
\tau: R^{r-i} f_{*} \Omega_{X / S}^{r} \rightarrow\left(R^{i} f_{*} \mathcal{O}_{X}\right)^{\vee},
$$

coming from the pairing

$$
R^{r-i} f_{*} \Omega_{X / S}^{r} \times R^{i} f_{*} \mathcal{O}_{X} \rightarrow R^{r} f_{*} \Omega_{X / S}^{r} \stackrel{\operatorname{Tr}}{\longrightarrow} \mathcal{O}_{S} .
$$

Let us now give the more precise formulation of Theorem 5.3.1

Theorem 5.3.3. Assumptions as in Theorem 5.3.1. For all $i$, the morphism

$$
R^{r-i} f_{*} \Omega_{X / S}^{r} \stackrel{(5.3 .1)}{\longrightarrow}\left(R^{i} f_{*} \mathcal{O}_{X}\right)^{\vee} \stackrel{(5.1 .1)}{\longrightarrow} H_{\mathrm{inv}}^{1}\left(\Phi^{i}(X / S)\right)
$$

extends to a surjective morphism of $D_{S}^{\prime}$-modules

$$
D_{S}^{\prime} \cdot \epsilon\left(R^{r-i} f_{*} \Omega_{X / S}^{r}\right) \rightarrow H_{\mathrm{inv}}^{1}\left(\Phi^{i}(X / S)\right) .
$$


Proof. Note that $R^{i} f_{*} \mathcal{O}_{X}=T \Phi^{i}(X / S)$, so that (5.1.1) makes sense. We may suppose that $S=\operatorname{Spec}(R)$ is affine. Furthermore, we may suppose $\Phi^{i}(X / S)=$ $\operatorname{Spf}\left(R\left[\left|x_{1}, \ldots, x_{d}\right|\right]\right)$ as formal schemes; in particular we already have a trivialization

$$
T \Phi^{i}(X / S)=\bigoplus_{j=1}^{d} \mathcal{O}_{S} e_{j}
$$

Denote by $e_{1}^{\vee}, \ldots, e_{d}^{\vee}$ the dual basis, set

$$
\begin{aligned}
& \alpha_{j}^{\vee}:=\tau^{-1}\left(e_{j}^{\vee}\right) \in \Gamma\left(R^{r-i} f_{*} \Omega_{X / S}^{r}\right), \\
& \omega_{j}:=\eta\left(e_{j}^{\vee}\right) \in \Gamma\left(\Omega_{\Phi^{i}(X / S) / S}^{1}\right), \quad \bar{\omega}_{j}:=\bar{\eta}\left(e_{j}^{\vee}\right) \in \Gamma\left(H^{1}\left(\Omega_{\Phi^{i}(X / S) / S}^{*}\right)\right) .
\end{aligned}
$$

If $P_{1}, \ldots, P_{d} \in \Gamma\left(D_{S}^{\prime}\right)$ satisfy

$$
\sum_{j=1}^{d} P_{j} \cdot \epsilon\left(\alpha_{j}^{\vee}\right)=0 \quad \text { in } \Gamma\left(H_{\mathrm{dR}}^{i}(X / S)\right),
$$

then we need to show that

$$
\sum_{j=1}^{d} P_{j} \cdot \bar{\omega}_{j}=0 \quad \text { in } \Gamma\left(H^{1}\left(\Omega_{\Phi^{i}(X / S) / S}^{*}\right)\right) .
$$

Our trivialization (5.3.5) induces an isomorphism

$$
\log : \Phi^{i}(X / S) \times_{S} S_{\mathbb{Q}} \cong \hat{\mathbb{G}}_{a, S_{\mathbb{Q}}}^{d},
$$

where $S_{\mathbb{Q}}$ is the base change to $\operatorname{Spec}(\mathbb{Q})$ (see A.2). Moreover, we have $d \log ^{*}\left(x_{j}\right)=$ $\omega_{j}$ (Section A.2.3). Write $\log ^{*}\left(x_{j}\right)=\sum_{I} g_{j, I} x^{I}$ with $g_{j, I} \in R \otimes_{\mathbb{Z}} \mathbb{Q}$. Then (5.3.6) is equivalent to

$$
f:=\sum_{I} \sum_{j=1}^{d} P_{j}\left(g_{j, I}\right) x^{I} \in R\left[\left|x_{1}, \ldots, x_{d}\right|\right] .
$$

Recall from (A.2.2) that log induces

$$
\log : \Phi^{i}(X / S)(R[|x|]) \rightarrow \hat{\mathbb{G}}_{a}((R \otimes \mathbb{Q})[|x|])^{d}=\bigoplus_{j=1}^{d} x \cdot(R \otimes \mathbb{Q})[|x|] .
$$

Moreover, the following diagram is commutative:

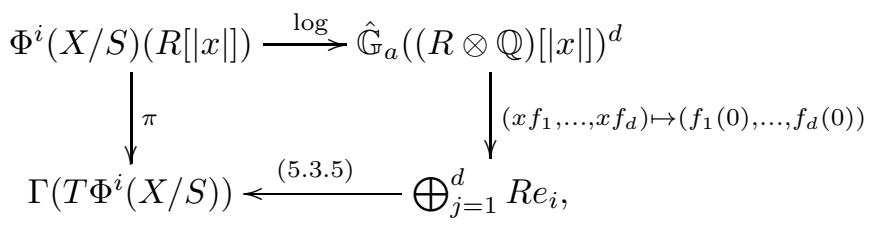

where $\pi$ is the projection. In view of Proposition 4.1.7 we have

$$
\Phi^{i}(X / S)(R[|x|])=H^{i}\left(X, \mathbb{W} \mathcal{O}_{X}\right)
$$

and $\pi$ corresponds to the projection $H^{i}\left(X, \mathbb{W} \mathcal{O}_{X}\right) \rightarrow H^{i}\left(X, \mathcal{O}_{X}\right)$ induced by the $\operatorname{map} \mathbb{W} \mathcal{O}_{X} \rightarrow \mathcal{O}_{X}$.

Let $\xi \in \Phi^{i}(X / S)(R[|x|])$ and write $\log (\xi)=:\left(\log (\xi)_{1}, \ldots, \log (\xi)_{d}\right)$. For $n \geq 1$ we obtain

$$
\pi\left(F_{n}(\xi)\right)=\sum_{j=1}^{d}\left(F_{n}\left(\log (\xi)_{j}\right)\right)(0) \cdot e_{j}
$$


because $\log$ is a morphism of $\mathbb{E}_{R}$-modules. Note that $\pi\left(F_{n}(\xi)\right) \in \Gamma\left(T \Phi^{i}(X / S)\right)$ implies $\left(F_{n}\left(\log (\xi)_{j}\right)\right)(0) \in R$. By construction, $\log (\xi)_{j}=\xi^{*} \log ^{*}\left(x_{j}\right) \in(R \otimes \mathbb{Q})[|x|]$, and we know that

$$
\left(F_{n}\left(\log (\xi)_{j}\right)\right)(0)=n \cdot\left(n \text {-th coefficient of } \xi^{*} \log ^{*}\left(x_{j}\right)\right) .
$$

A theorem of Stienstra 5.4.1 implies

$$
\sum_{j=1}^{d} P_{j} \cdot\left(F_{n}\left(\log (\xi)_{j}\right)\right)(0) \in n R
$$

hence

$$
\sum_{j=1}^{d} P_{j} \cdot\left(n \text {-th coefficient of } \xi^{*} \log ^{*}\left(x_{j}\right)\right) \in R \quad \text { for all } n .
$$

Now suppose that $\xi^{*}\left(x_{j}\right) \in \mathbb{Z}[|x|]$ for every $j=1, \ldots, d$ (where the $x_{j}$ are now the coordinates from $\left.\Phi^{i}(X / S)=\operatorname{Spf}\left(R\left[\left|x_{1}, \ldots, x_{d}\right|\right]\right)\right)$. Then (5.3.7) implies that $\xi^{*}(f) \in R[|x|]$. In view of Lemma $\left[5.3 .4\right.$ we finally obtain $f \in R\left[\left|x_{1}, \ldots, x_{d}\right|\right]$.

Lemma 5.3.4. Let $R$ be flat over $\mathbb{Z}$. Let $f \in\left(R \otimes_{\mathbb{Z}} \mathbb{Q}\right)\left[\left|x_{1}, \ldots, x_{d}\right|\right]$. Suppose that $\xi^{*}(f) \in R[|x|]$ for all $\xi: \operatorname{Spf}(R[|x|]) \rightarrow \operatorname{Spf}\left(R\left[\left|x_{1}, \ldots, x_{d}\right|\right]\right)$ such that $\xi^{*}\left(x_{j}\right) \in \mathbb{Z}[|x|]$ for all $j$. Then $f \in R\left[\left|x_{1}, \ldots, x_{d}\right|\right]$.

Proof. We prove by induction on $d$. The case $d=1$ is trivial. By subtracting an element in $R\left[\left|x_{1}, \ldots, x_{d}\right|\right]$ we may assume that

$$
f=x_{d}^{m} \cdot \sum_{i=0}^{\infty} f_{i}\left(x_{0}, \ldots, x_{d-1}\right) x_{d}^{i}
$$

and $f_{0} \notin R\left[\left|x_{0}, \ldots, x_{d-1}\right|\right]$. For every $\xi^{\prime}: \operatorname{Spf}(R[|x|]) \rightarrow \operatorname{Spf}\left(R\left[\left|x_{1}, \ldots, x_{d-1}\right|\right]\right)$ such that $\xi^{*}\left(x_{j}\right) \in \mathbb{Z}[|x|]$ for all $j$, and every integer $n \geq 1$, we define $\xi$ by $\xi^{*}\left(x_{j}\right)=$ $\xi^{* *}\left(x_{j}\right)$ if $j<d$ and $\xi^{*}\left(x_{d}\right)=x_{d}^{n}$. Let $n$ tend to $\infty$ in order to obtain $\xi^{\prime *}\left(f_{0}\right) \in$ $R\left[\left|x_{1}, \ldots, x_{d-1}\right|\right]$.

As in Corollary 5.3 .2 we obtain the following statement.

Corollary 5.3.5. Let $f: X \rightarrow S$ be a smooth, projective morphism of relative dimension $r$. Suppose that $S$ is smooth over $\operatorname{Spec}(\mathbb{Z})$ and suppose that $R^{j} f_{*} \mathcal{O}_{X}$ is locally free for all $j$. Then, for all $i$, the sheaf $H_{\mathrm{inv}}^{1}\left(\Phi^{i}(X / S)\right) \otimes_{\mathcal{O}_{S}} \mathcal{O}_{S_{\mathbb{Q}}}$ is a quotient of the submodule $D_{S_{\mathbb{Q}}} \cdot R^{r-i} f_{*} \Omega_{X_{\mathbb{Q}} / S_{\mathbb{Q}}}^{r}$ of $H_{\mathrm{dR}}^{2 r-i}\left(X_{\mathbb{Q}} / S_{\mathbb{Q}}\right)$.

Remark 5.3.6. We don't know examples where the quotient map

$$
D_{S_{\mathbb{Q}}} \cdot R^{r-i} f_{*} \Omega_{X_{\mathbb{Q}} / S_{\mathbb{Q}}}^{r} \rightarrow H_{\mathrm{inv}}^{1}\left(\Phi^{i}(X / S)\right) \otimes_{\mathcal{O}_{S}} \mathcal{O}_{S_{\mathbb{Q}}}
$$

is not an isomorphism.

5.4. In the following we recall the theorem of Stienstra that is used in the proof of Theorem 5.3.3. Stienstra proved it in [Sti91, Theorem 4.6] by using his definition of the big de Rham-Witt complex. For the convenience of the reader we will recall the proof and work with the big de Rham-Witt complex introduced in Hes].

Theorem 5.4.1 (Sti91, Theorem 4.6]). Let $f: X \rightarrow S$ be a smooth, projective morphism of relative dimension $r$. Suppose that $S=\operatorname{Spec}(R)$ is smooth over $\operatorname{Spec}(\mathbb{Z})$ and suppose that $R^{j} f_{*} \Omega_{X / S}^{i}$ is free for all $i, j$. Fix an integer $m \geq$ 0 . Take a basis $\omega_{1}, \ldots, \omega_{h}$ of $H^{m}\left(X, \mathcal{O}_{X}\right)$. Let $\omega_{1}^{\vee}, \ldots, \omega_{h}^{\vee}$ be the dual basis of 
$H^{r-m}\left(X, \Omega_{X / S}^{r}\right)$. Take $\xi \in H^{m}\left(X, \mathbb{W O}_{X}\right)$ and define for every positive integer $n$ the elements $B_{n, 1}, \ldots, B_{n, h} \in R$ by

$$
\pi\left(F_{n} \xi\right)=\sum_{j=1}^{h} B_{n, j} \omega_{j}
$$

where $\pi: H^{m}\left(X, \mathbb{W} \mathcal{O}_{X}\right) \rightarrow H^{m}\left(X, \mathcal{O}_{X}\right)$ is the projection.

Suppose $P_{1}, \ldots, P_{h} \in \Gamma\left(D_{S}^{\prime}\right)$ are such that

$$
\sum_{j=1}^{h} P_{j} \omega_{j}^{\vee}=0 \quad \text { in } H^{2 r-m}\left(X, \Omega_{X / S}^{*}\right),
$$

then

$$
\sum_{j=1}^{h} P_{j} B_{n, j}=0 \quad \bmod n .
$$

Proof. For a truncation set $T$, let $\mathbb{W}_{T} \Omega_{X}^{*}$ be the big de-Rham Witt complex defined in $\mathrm{Hes}$. We will only need a relative version: set

$$
\mathbb{W} \Omega_{X / S}^{*}:={\underset{\lim }{T}}_{T} \mathbb{W}_{T} \Omega_{X}^{*} /\left(f^{-1} \mathbb{W}_{T} \Omega_{S}^{1} \cdot \mathbb{W}_{T} \Omega_{X}^{*}\right),
$$

where $T$ runs over all finite truncation sets. We denote by $\tau$ the projection $\mathbb{W} \Omega_{X / S}^{*} \rightarrow \Omega_{X / S}^{*}$, it is a morphism of differential graded algebras. For all $n \geq 1$ we have the Frobenius $F_{n}: \mathbb{W} \Omega_{X / S}^{*} \rightarrow \mathbb{W} \Omega_{X / S}^{*}$ satisfying $d F_{n}=n \cdot F_{n} d$. Moreover, there is a natural morphism

$$
\eta_{X / S}: \mathbb{W} \mathcal{O}_{X} \rightarrow \mathbb{W} \Omega_{X / S}^{*}
$$

that identifies $\mathbb{W} \Omega_{X / S}^{0}$ with $\mathbb{W} \mathcal{O}_{X}$, but $\eta_{X / S}$ is in general not a morphism of complexes.

For all integers $n \geq 1$, we set

$$
\mathbb{Z} / n \otimes \mathbb{W} \Omega_{X / S}^{*}:=\operatorname{coker}\left(\mathbb{W} \Omega_{X / S}^{*} \stackrel{n \cdot}{\longrightarrow} \mathbb{W} \Omega_{X / S}^{*}\right),
$$

and similarly we define $\mathbb{Z} / n \otimes \Omega_{X / S}^{*}$; we denote by $\epsilon_{n}$ the quotient map $\mathbb{W} \Omega_{X / S}^{*} \rightarrow$ $\mathbb{Z} / n \otimes \mathbb{W} \Omega_{X / S}^{*}$. The rule $d F_{n}=n F_{n} d$ implies that we obtain a commutative diagram of morphisms of complexes

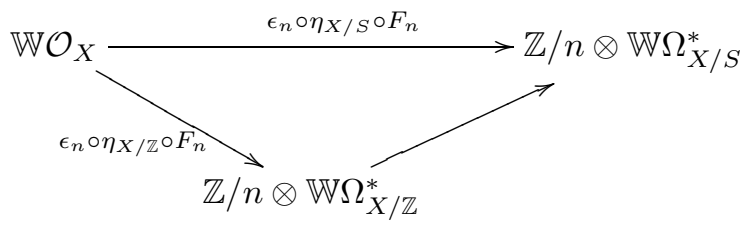

We set

$$
\zeta_{n}:=\left(\tau \circ \epsilon_{n} \circ \eta_{X / S} \circ F_{n}\right)(\xi) \in H^{m}\left(X, \mathbb{Z} / n \otimes \Omega_{X / S}^{*}\right) ;
$$

note that $H^{m}\left(X, \mathbb{Z} / n \otimes \Omega_{X / S}^{*}\right)=H_{\mathrm{dR}}^{m}(X / S) \otimes_{R} R / n$, because the Hodge to de Rham spectral sequence degenerates at $E_{1}$ and hence $H_{\mathrm{dR}}^{*}(X / S)$ is a free $R$-module. Recall that $H^{r-m}\left(X, \Omega_{X / S}^{r}\right) \subset H_{\mathrm{dR}}^{2 r-m}(X / S)$, as a next step we need to prove

$$
\left\langle\zeta_{n}, \omega_{j}^{\vee}\right\rangle=B_{n, j} \bmod n R,
$$


via the pairing

$$
\langle., .\rangle: H_{\mathrm{dR}}^{m}(X / S) \times H_{\mathrm{dR}}^{2 r-m}(X / S) \rightarrow H_{\mathrm{dR}}^{2 r}(X / S)=H^{r}\left(X, \Omega_{X / S}^{r}\right) \stackrel{\operatorname{Tr}}{\longrightarrow} R .
$$

Indeed, we have

$$
\left\langle\zeta, \omega_{j}^{\vee}\right\rangle=\left(q(\zeta), \omega_{j}^{\vee}\right) \quad \text { for all } \zeta \in H_{\mathrm{dR}}^{m}(X / S),
$$

with $q: H_{\mathrm{dR}}^{m}(X / S) \rightarrow H^{m}\left(X, \mathcal{O}_{X}\right)$ induced by the projection $\Omega_{X / S}^{*} \rightarrow \mathcal{O}_{X}$, and $(.,$.$) is the Grothendieck duality pairing (5.3.2). Now the equality q\left(\zeta_{n}\right)=\pi\left(F_{n} \xi\right)$ modulo $n R$ implies (5.4.1).

Since $\zeta_{n}$ is the image of the class $\left(\tau \circ \epsilon_{n} \circ \eta_{X / \mathbb{Z}} \circ F_{n}\right)(\xi) \in H^{m}\left(X, \mathbb{Z} / n \otimes \Omega_{X / \mathbb{Z}}^{*}\right)$, it is horizontal for the Gauss-Manin connection. Therefore $D\left(\zeta_{n}\right)=0$ for all derivations $D$ of $R$. From the compatibility of $\langle.,$.$\rangle with the Gauss-Manin connection we get$

$$
D\left(B_{n, j}\right)=\left\langle D\left(\zeta_{n}\right), \omega_{j}^{\vee}\right\rangle+\left\langle\zeta_{n}, D\left(\omega_{j}^{\vee}\right)\right\rangle=\left\langle\zeta_{n}, D\left(\omega_{j}^{\vee}\right)\right\rangle \bmod n R
$$

for all derivations. This implies the theorem.

Example 5.4.2. The logarithm of the formal group $\Phi^{n-r}(X / R)$ attached to a complete intersection $X \subset \mathbb{P}_{R}^{n}$ of codimension $r$ has been computed by Stienstra Sti87.

The classical example for Theorem 5.3.1 is the Legendre family $X=\left\{z y^{2}-\right.$ $x(x-z)(x-\lambda z)=0\} \subset \mathbb{P}_{R}^{2}$ of elliptic curves, with $R=\mathbb{Z}[\lambda]\left[\frac{1}{2 \lambda(1-\lambda)}\right]$. An invariant one-form of $\Phi^{1}(X / R)$ is given by

$$
\omega=\sum_{\substack{n=0 \\
n \text { even }}}^{\infty}\left(\begin{array}{l}
n \\
\frac{n}{2}
\end{array}\right) \sum_{k=0}^{\frac{n}{2}}\left(\begin{array}{l}
\frac{n}{2} \\
k
\end{array}\right)^{2} \lambda^{k} x^{n} d x
$$

with the coordinate $x$ from Sti87. $\left(\Phi^{1}(X / R)\right.$ is one dimensional). As $D_{R^{\prime}}^{\prime}$-module, $H_{\mathrm{dR}}^{1}(X / R)$ is annihilated by

$$
D:=\lambda(1-\lambda)\left(\frac{d}{d \lambda}\right)^{2}+(1-2 \lambda) \frac{d}{d \lambda}-\frac{1}{4}
$$

Kat84. Since $\left(\begin{array}{c}n \\ \frac{n}{2}\end{array}\right) \equiv \pm 1 \bmod n+1$, Theorem 5.3.1 implies that

$$
D\left(\sum_{k=0}^{\frac{n}{2}}\left(\begin{array}{l}
\frac{n}{2} \\
k
\end{array}\right)^{2} \lambda^{k}\right)=0 \quad \bmod n+1,
$$

which the reader may prove easily by a direct computation (see Kat84). The sequence $\left(\sum_{k=0}^{\frac{n}{2}}\left(\begin{array}{c}\frac{n}{2} \\ k\end{array}\right)^{2} \lambda^{k}\right)_{n+1}$ forms an element in

$$
F \in \lim _{\substack{n+1 \\ n \text { even }}} \mathbb{Z} /(n+1)[\lambda] \subset\left(\prod_{p \neq 2} \mathbb{Z}_{p}\right)[|\lambda|]
$$

(the projective system is ordered by division) that satisfies $D(F)=0$ and $F(0)=1$; thus it is given by the hypergeometric series $F={ }_{2} F_{1}\left(\frac{1}{2}, \frac{1}{2} ; 1, \lambda\right)$. Since $F$ suffices to reconstruct the operator $D$, the quotient morphism from Corollary 5.3.5 is an isomorphism in this case.

The analogous statements can also be proved for the Dwork family $\left\{\sum_{i=0}^{m} x_{i}^{m+1}-\right.$ $\left.(m+1) \lambda x_{0} \cdots x_{m}=0\right\} \subset \mathbb{P}_{R}^{m}$ of Calabi-Yau varieties (see Yu09]). 


\section{Appendix A. Commutative formal Lie groups}

In this section we recall some basic facts in the theory of commutative formal Lie groups, that we need in the main text of the paper.

\section{A.1. Invariant 1-forms.}

A.1.1. Let $S$ be a scheme and $\mathfrak{X}$ be a commutative formal Lie group over $S$ (see 1.5).

Definition A.1.2. A 1-form $\omega \in \Omega_{\mathfrak{X} / S}$ is called invariant if

$$
m^{*}(\omega)=\operatorname{pr}_{1}^{*}(\omega)+\operatorname{pr}_{2}^{*}(\omega),
$$

where $m: \mathfrak{X} \times{ }_{S} \mathfrak{X} \rightarrow \mathfrak{X}$ is the multiplication and $\operatorname{pr}_{i}$ are the projections.

For example, $d x$ is an invariant 1 -form for $\hat{\mathbb{G}}_{a}$, and $d x /(1-x)$ is an invariant 1-form for $\hat{\mathbb{G}}_{m}$ in the coordinates from 1.1. Obviously, the invariant 1-forms form a $\mathcal{O}_{S}$-submodule of $\Omega_{\mathfrak{X} / S}$.

In 1.5 we introduced the tangent space of $\mathfrak{X}$ by

$$
T \mathfrak{X}(U):=\operatorname{ker}\left(\mathfrak{X}\left(\operatorname{Spec}\left(\mathcal{O}_{U}[\epsilon] / \epsilon^{2}\right)\right) \rightarrow \mathfrak{X}(U)\right),
$$

where $U \rightarrow \operatorname{Spec}\left(\mathcal{O}_{U}[\epsilon] / \epsilon^{2}\right)$ is the morphism over $U$ given by $\epsilon \mapsto 0$. Given $\xi \epsilon$ $T \mathfrak{X}(U)$, we can define a derivation $D_{\xi}: \Gamma\left(U, \mathcal{O}_{\mathfrak{X}}\right) \rightarrow \Gamma\left(U, \mathcal{O}_{S}\right)$ by

$$
D_{\xi}(f) \epsilon=\xi^{*}(f)-f(0) .
$$

Here and in the following we consider $\mathcal{O}_{S}$ as an $\mathcal{O}_{\mathfrak{X}}$-module via the zero section $f \mapsto f(0)$. This induces an isomorphism of $\mathcal{O}_{S}$-modules

$$
T \mathfrak{X} \rightarrow \operatorname{Der}_{\mathcal{O}_{S}}\left(\mathcal{O}_{\mathfrak{X}}, \mathcal{O}_{S}\right)=\operatorname{Hom}_{\mathcal{O}_{\mathfrak{X}}}\left(\Omega_{\mathfrak{X} / S}, \mathcal{O}_{S}\right) .
$$

Proposition A.1.3. There is a unique morphism of $\mathcal{O}_{S}$-modules

$$
\eta: T \mathfrak{X}^{\vee} \rightarrow \Omega_{\mathfrak{X} / S},
$$

satisfying the following properties:

(1) The morphism $\eta$ induces an isomorphism between $T \mathfrak{X}^{\vee}$ and the invariant 1-forms.

(2) The diagram

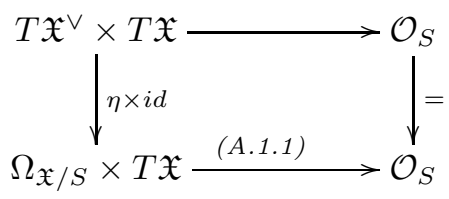

is commutative.

Proof. [Zin84, 1.19]

If $\mathfrak{X}=\operatorname{Spf}\left(\mathcal{O}_{S}\left[\left|x_{1}, \ldots, x_{d}\right|\right]\right)$ as formal scheme over $S$ then

$$
\Omega_{\mathfrak{X} / S} \rightarrow \mathcal{O}_{S}^{d}, \quad \sum_{i=1}^{d} f_{i} d x_{i} \mapsto\left(f_{1}(0), \ldots, f_{d}(0)\right),
$$

induces an isomorphism between the invariant 1-forms and $\mathcal{O}_{S}^{d}$.

Lemma A.1.4. Let $S$ be a scheme that is flat over $\operatorname{Spec}(\mathbb{Z})$. Let $\mathfrak{X}$ be a commutative formal Lie group over $S$. Then the invariant 1-forms are closed. 
Proof. We may suppose $S=\operatorname{Spec}(R)$. Denoting by $S \otimes_{\mathbb{Z}} \mathbb{Q}$ the base change to $\operatorname{Spec}(\mathbb{Q})$, we have $\Gamma\left(S, \Omega_{\mathfrak{X} / R}^{2}\right) \subset \Gamma\left(S \otimes_{\mathbb{Z}} \mathbb{Q}, \Omega_{\mathfrak{X} \otimes_{\mathbb{Z}} \mathbb{Q} / R \otimes_{\mathbb{Z}}}^{2}\right)$. Since $\mathfrak{X} \otimes_{\mathbb{Z}} \mathbb{Q}$ is isomorphic to the formal completion of a vector bundle (Section A.2) we are reduced to the obvious case $\hat{\mathbb{G}}_{a}$.

\section{A.2. Logarithms and formal groups in characteristic zero.}

A.2.1. Let $S$ be a scheme. Recall from Section 1.5 that we have a functor (A.2.1)

$T$ : (Comm. formal Lie groups over $S) \rightarrow$ (loc. free sheaves on $S$ of finite rank),

that assigns to a group its tangent space (or Lie algebra).

Proposition A.2.2. Suppose that $S$ is a scheme over $\operatorname{Spec}(\mathbb{Q})$. Then $T$ (A.2.1) is an equivalence of categories.

Proof. [Zin84, 4.7]

For every locally free sheaf $\mathcal{E}$ of finite rank on $S$ we have the associated vector bundle

$$
V(\mathcal{E}):=\operatorname{Spec}\left(\operatorname{Sym}^{*} \mathcal{E}^{\vee}\right) .
$$

The formal completion $\hat{V}(\mathcal{E})$ yields a commutative formal Lie group with tangent space $\mathcal{E}$. If $S$ is a scheme over $\mathbb{Q}$, then for every commutative formal Lie group $\mathfrak{X}$ there exists a unique isomorphism of formal groups

$$
\mathfrak{X} \rightarrow \hat{V}(T \mathfrak{X}),
$$

such that the induced map on the tangent spaces is the identity; the functor $\hat{V}$ is inverse to $T$. For every morphism $\phi: T \mathfrak{X} \rightarrow \mathcal{E}$ we will denote by

$$
\log _{\phi}: \mathfrak{X} \rightarrow \hat{V}(\mathcal{E})
$$

the unique morphism over $\phi$.

In general we identify $\hat{V}\left(\mathcal{O}_{S}^{d}\right)$ with $\hat{\mathbb{G}}_{a}^{d}$. A basic example is $\mathfrak{X}=\hat{\mathbb{G}}_{m}, \mathcal{E}=\mathcal{O}_{S}$, and $\phi$ coming from the coordinate of $\hat{\mathbb{G}}_{m}$ in 1.1 Then $\log _{\phi}: \hat{\mathbb{G}}_{m} \rightarrow \hat{\mathbb{G}}_{a}$ is given by $\log _{\phi}^{*}(x)=\sum_{k=1}^{\infty} \frac{x^{k}}{k}$.

A.2.3. Again, suppose that $S$ is a scheme over $\operatorname{Spec}(\mathbb{Q})$. The canonical morphism $\mathfrak{X} \rightarrow \hat{V}(T \mathfrak{X})$ can be constructed as follows. By Proposition A.1.3 we have a canonical morphism $\eta: T \mathfrak{X}^{\vee} \rightarrow \Omega_{\mathfrak{X} / S}$ that induces an isomorphism with the invariant forms. Since invariant forms are closed (Lemma A.1.4) we obtain a morphism of $\mathcal{O}_{S}$-modules

$$
d^{-1} \circ \eta: T \mathfrak{X}^{\vee} \rightarrow \operatorname{ker}\left(0^{*}: \mathcal{O}_{\mathfrak{X}} \rightarrow \mathcal{O}_{S}\right) .
$$

This morphism induces $\mathfrak{X} \rightarrow \hat{V}(T \mathfrak{X})$.

A.2.4. Let $S=\operatorname{Spec}(R)$ be flat over $\operatorname{Spec}(\mathbb{Z})$. Let $\mathfrak{X}$ be a commutative formal Lie group. Suppose that $\mathfrak{X}=\operatorname{Spf}\left(R\left[\left|x_{1}, \ldots, x_{d}\right|\right]\right)$ as formal schemes and that the zero is defined by $x_{i} \mapsto 0$. We denote by $\mathfrak{X}_{\mathbb{Q}}$ the base change to $S_{\mathbb{Q}}:=S \times_{\operatorname{Spec}(\mathbb{Z})} \operatorname{Spec}(\mathbb{Q})$.

Since $T \mathfrak{X}$ comes equipped with a trivialization, we have a canonical map

$$
\mathfrak{X}(R[|x|]) \rightarrow \mathfrak{X}_{\mathbb{Q}}((R \otimes \mathbb{Q})[|x|]) \stackrel{\log }{\longrightarrow} \hat{\mathbb{G}}_{a}^{d}((R \otimes \mathbb{Q})[|x|])=\bigoplus_{i=1}^{d} x \cdot(R \otimes \mathbb{Q})[|x|] .
$$




\section{REFERENCES}

[AM77] M. Artin and B. Mazur. Formal groups arising from algebraic varieties. Ann. Sci. École Norm. Sup. (4), 10(1):87-131, 1977.

[BBE07] Pierre Berthelot, Spencer Bloch, and Hélène Esnault. On Witt vector cohomology for singular varieties. Compos. Math., 143(2):363-392, 2007.

[Hes] Lars Hesselholt. The big de Rham-Witt complex. arXiv:1006.3125v1.

[HM01] Lars Hesselholt and Ib Madsen. On the $K$-theory of nilpotent endomorphisms. In Homotopy methods in algebraic topology (Boulder, CO, 1999), volume 271 of Contemp. Math., pages 127-140. Amer. Math. Soc., Providence, RI, 2001.

[Hon70] Taira Honda. On the theory of commutative formal groups. J. Math. Soc. Japan, 22:213246, 1970.

[Kat84] Nicholas M. Katz. Expansion-coefficients as approximate solution of differential equations. Astérisque, (119-120):6, 183-189, 1984. p-adic cohomology.

[KO68] Nicholas M. Katz and Tadao Oda. On the differentiation of de Rham cohomology classes with respect to parameters. J. Math. Kyoto Univ., 8:199-213, 1968.

[Sti87] Jan Stienstra. Formal group laws arising from algebraic varieties. Amer. J. Math., 109(5):907-925, 1987.

[Sti91] Jan Stienstra. The generalized de Rham-Witt complex and congruence differential equations. In Arithmetic algebraic geometry (Texel, 1989), volume 89 of Progr. Math., pages 337-358. Birkhäuser Boston, Boston, MA, 1991.

[Yu09] Jeng-Daw Yu. Variation of the unit root along the Dwork family of Calabi-Yau varieties. Math. Ann., 343(1):53-78, 2009.

[Zin84] Thomas Zink. Cartiertheorie kommutativer formaler Gruppen, volume 68 of TeubnerTexte zur Mathematik [Teubner Texts in Mathematics]. BSB B. G. Teubner Verlagsgesellschaft, Leipzig, 1984. With English, French and Russian summaries.

Fachbereich Mathematik, Universität Duisburg-Essen, 45117 Essen, Germany

E-mail address: a.chatzistamatiou@uni-due.de 